\title{
Factors Contributing to Delayed Health Seeking Behaviours Among Adolescents
}

\author{
Ripfumelo F. Mboweni ${ }^{1}$ \& Gsakani O. Sumbane ${ }^{1}$ \\ ${ }^{1}$ School of Health Care Sciences, University of Limpopo, Polokwane, South Africa \\ Correspondence: Gsakani O. Sumbane, Department of Nursing Science, School of Health Care Sciences, \\ University of Limpopo, Private Bag X1106, Sovenga 0727, Polokwane, South Africa. Tel: 27-082-562-0888; \\ 27-015-268-4241. E-mail: gsakani.sumbane@ul.ac.za
}

Received: September 10, 2019 Accepted: October 4, 2019 Online Published: November 6, 2019

doi:10.5539/gjhs.v11n13p67

URL: https://doi.org/10.5539/gjhs.v11n13p67

\begin{abstract}
Background: Most adolescents for various reasons fail to go and seek for health care regarding health risks and challenges until they are faced with the complications. The socio-cultural belief concerning the causes of illnesses and its characteristic has correlation with the treatment seeking behaviour of an individual.

Objectives: The study explored and described factors contributing to delayed health-seeking behaviours amongst adolescents in Kutlwanong clinic, North West Province.

Method: This was a qualitative study designed using a phenomenological approach. Individual semi-structured in-depth interviews were audiotaped and transcribed verbatim. 8 Steps of Tesch's inductive, descriptive open coding technique by Creswell was used followed by independent coding. Purposive sampling recruited fourteen participants aged 15-18 years. Trustworthiness criteria adhered to were credibility, dependability, transferability, and confirmability. Ethical clearance was sought with the University of Limpopo.
\end{abstract}

Results: Factors that negatively impact adolescents' health-seeking behavior include the negative attitude of nurses and community, influenced by peers, shortage of medicine, lack of services throughout weekends, long queues, waiting times, distance, and lack of knowledge. Family planning and antenatal care were the common reason for seeking health care.

Conclusion: Being aware of factors contributing to delay health-seeking behaviours will facilitate with setting future priorities to guard the health, well-being, and dignity of adolescents. The impacts of youth programs must be monitored on a regular basis to assess the needs and capabilities of specific facilities. Clinic manager to ensure compliance with the national core standards to meet adolescent's immediate expectations.

Keywords: adolescents, delayed, health-seeking behaviour

\section{Introduction}

Health seeking behaviours play a significant role within the improvement of the healthcare status of the nation (Jim, 2010). As a result, it is considered as an indicator of future population health, social and economic development as explained by Channamma (2017). It has become an instrument for considering how people interact with the health care systems. The socio-cultural belief concerning the causes of illnesses and its characteristic has correlation with the treatment seeking behaviour of an individual. Health-seeking behaviour in this study is defined as the approaches in which adolescents interpret health problems and action taken to remedy these problems. It settled inside the broader plan of health care utilization, which encompasses activities undertaken to prevent and curing health issues, maintain physiological state and well-being, or obtaining data regarding one's health status and prognosis (Latunji \& Akinyemi, 2018).

Adolescents vary in their readiness to seek help from health care services. Some go without a delay others only when in an advanced state of a health problem as mentioned by (Channamma, 2017). Prior studies in South Africa indicated various health problems encountered by adolescents these include unintended pregnancies of approximately 35\%, HIV\&AIDS, as a currently a major concern (van der Hoeven, Kruger, \& Greeff, 2012), reproductive and sexual health (Otwombe, Dietrich, Laher, Hornschuh, Nkala, Chimoyi, \& Miller, 2015). Other general concerns about adolescent were accidental injuries, mental disorders (e.g. depression) (Ybarra, Emenyonu, Nansera, Kiwanuka, \& Bangsberg, 2007), substance abuse and malnutrition (James et al., 2018; Channamma, 
2017). Adolescents in Soweto reported seeking health for health problems such as condom breakage, flu-like symptoms, and reproductive health (Otwombe et al., 2015). With all these health issues and risks that adolescents are faced with, most of them fail to go and seek health care for numerous reasons until they are faced with the complications. Adolescents are content with the established order of their lives rather than looking for health care services when perceiving ill health. Unfortunately, not obtaining help will result in worsening of the condition (Fertman, Delgado, \& Tarasevich, 2013).

To overcome issues the National Department of Health in South Africa developed various mechanisms and programs to boost adolescent's health, as explained within the National adolescents and Youth Health Policy (National Department of Health, 2017). These embrace; (i) Adolescent and Youth Friendly Services (AYFS) that may be a standards-driven approach to boost quality of look after adolescents and youth (Otwombe et al., 2015). (ii) Integrated Faculty Health Programme: focuses on addressing learner's health issues; (iii) Ideal clinics: clinics with smart infrastructure, adequate employees, drugs and supplies (iii) B-Wise: a program to empower adolescents to make decisions based on correct information; and (iv) She Conquers Campaign: A youth-led campaign to handle the main problems encountered by young women nowadays in SA. Despite the facilities having the essential parts for general service delivery in situ, adolescent-specific service provision reported being lacking as mentioned by (James et al., 2018). Ajaegbu and Ubochi, (2016) mentioned that the timely intervention on major health issues can cut back the mortality and morbidity rates, however this is often difficult in African countries wherever the sick often have access to formal health care services and most deaths occur.

Addressing adolescent health-seeking behaviour is very important and therefore the opinion that adolescence being a time of transitions for both physical and social or emotional, that have implications for health (James et al., 2018; Fertman et al., 2013). Adolescents have specific health issues and developmental needs that vary from those of children and adult. The causes of pathological state in adolescents are principally psychosocial instead of biological (Ajaegbu \& Ubochi, 2016).The study can assist within the identification of the factors that delayed utilization of health care services by adolescents. Two major frameworks are planned to clarify the utilization of health care services by the individual from the behavioural attributes (Channamma, 2017). These are Andersen and Newman model (1973) and therefore the Kroeger's model (1983).

Andersen and Newman model (1973) believed that associate individual's access to and use of health services is taken into account to be a operate of three factors. These include predisposing factors that are the socio-cultural characteristics of people that exist prior ill, health, academic background, attitude, values, age, and gender. Enabling factors which include the provision aspect of getting care like accessibility, cost, waiting time and travel. Needs factors that embody the foremost immediate explanation for health service use, from practical and health issues that generate the requirement for health care services (Tesfaye, Chojenta, Smith, \& Loxton, 2018). Similarly, Kroeger's model (1983) consists of three characteristics that influence the use of healthcare-seeking behaviour, which incorporates the subject, health service and perceived illness characteristics. The subject characteristics embody age, sex, house size that is analogous to the predisposing factors, and health services characteristics embody accessibility, acceptableness, a value that is additionally kind of like the enabling factors of Andersen and Newman model (1973). The characteristics of perceived illness contain whether or not the condition is chronic or acute, severity and advantages of the treatment (Channamma, 2017).

Based on the frameworks healthcare-seeking behaviour could be an advanced outcome of many factors operating at individual, family and community level (van der Hoeven et al., 2012). Research on health-seeking behaviours among adolescents within the North West province of SA is restricted. To assist address this gap, we describe here the factors contributing to delay health-seeking behaviours amongst adolescents at Kutlwanong clinic within the rural community, of Cyferskuil, North West province, SA.

\section{Material and Methods}

\subsection{Research Design}

This was a qualitative study designed using a phenomenological approach in the selected clinic of North West Province, (South Africa) SA.

\subsection{Ethical Issues}

Ethical clearance was obtained from Turfloop Research Ethics Committee at the University of Limpopo (TREC/65/2018: PG), permission to collect data was obtained from the Department of Health in North West province, South Africa and from the Kutlwanong clinic operational manager. Informed consent was obtained and forms were signed before participation. Confidentiality and anonymity were maintained throughout the study. 


\subsection{Setting}

The study was conducted at Kutlwanong clinic which is situated in Cyferskuil village, Moretele sub-district, Bojanala district, North West province, South Africa. It renders health services to the community of Cyferskuil and the surrounding communities. The majority of clinic patients are black African. The clinic has a high number of pregnant adolescent's late Basic Antenatal Care (BANC) bookings. According to the clinic register most of the adolescent's book for BANC when they are above 14 weeks. Other adolescents come to the clinic being on advanced stage for sexually transmitted infections.

\subsection{Identification of Participants}

Purposive sampling recruited 12 participants (10 females and 2 males aged 15-18 years). All adolescents with delayed health-seeking behaviours were purposively sampled during a consultation at the selected clinic. Non-pregnant, pregnant, and healthy were eligible to participate in this study. Interested participants were invited to take part in the study. Appointments were made for data collection. Adolescents who were not stable and do not show a delay in help-seeking behaviours did not form part of the study.

\subsection{Procedure}

A semi-structured interview guide was developed and piloted with adolescents. A central question asked to every participantis as follows: "Kindly describe in details factors that are preventing you from consulting early when you need health care services". Clarity seeking questions followed after the participants' responses to the central questions. Data were collected by the principal investigators after ethical permission was obtained. The 12 individual interviews provided rich descriptions. All interviews were conducted in English, were audio-recorded with the permission of the participants and lasted 30-60 min. The interviews were conducted at the clinic in a private room. Field notes were taken to capture non-verbal cues.

\subsection{Data Analysis}

The audio recordings of the individual semi-structured in-depth interviews were transcribed verbatim. Eight Steps of Tesch's inductive, descriptive open coding qualitative data analysis technique as described by Creswell (2014) was used to analyze data. Data was also submitted to an independent coder who also used eight steps of Tesch's open coding method of qualitative data analysis as described by Creswell (2014). A consensus meeting was set between the researcher and the independent coder in order to discuss and agree on final themes and sub-themes based on the ones which emerged when analyzing independently.

Table 1 depicts the final themes and sub-themes which were agreed upon by the researcher and independent coder which serves as the findings of the study. Two themes and eight sub-themes emerged as illustrated in Table 1. Verbatim comments from the 12 participants are reported below, for example, P1=Participants number one.

\section{Result and Discussion}

Table 1. Themes and subthemes

\begin{tabular}{ll}
\hline Themes & Sub-themes \\
\hline & 1.1 The negative attitude of nurses towards adolescents \\
& 1.2 The negative attitude of the community towards adolescents. \\
& 1.3 Attitude and influenced by peers. \\
1. Socio-cultural characteristics of individuals & 1.4 Knowledge of adolescents concerning and towards health care \\
& systems \\
\hline 2. The logistical aspects of obtaining care & 2.1 Shortage of treatment and lack of services throughout weekends \\
& 2.2 Long queues and waiting time \\
& 2.3 Distance and family responsibilities \\
& 2.4 Common reasons for seeking health \\
\hline
\end{tabular}

\subsection{Theme 1: Socio-Cultural Characteristics of Individuals}

The first theme identified was that the attitude and influences of nurses, community, and peers delay the participants to seek health when they are sick. The nurse's attitudes towards the adolescents were perceived as the 
main factor, in fact, all participants highlighted it.

\subsubsection{The Attitude of Nurses Towards Adolescents}

Several adolescents explained that the attitude of nurses plays a role in the attendance of clinic, as a results nurse's gossip about the clients' diagnosis and pass the information to the community, breach confidentiality between the nurse and the client, shout and treat adolescents badly. The participants additionally expressed that nurses hurt them with words. "Nurses will shout at you, hurting with words, treat people badly; they are the ones that start gossiping in the clinic and pass the gossip to other community members". (P3)

"There are some nurses here at the clinic, they make us hate coming to the clinic". (P6)

One adolescent declares that she rather have complications or die than to listen to individuals gossip mongering concerning her. As a participant noted:

"The gossip things are serious I'm too terrified of the way people speak, I'd rather die than people spreading rumours about me". (P2)

Furthermore, adolescents were found not being comfortable to share their problems with older nurses as a result of they believed that they are judgmental. As proven by "once I get inside the consulting room and I am being assisted by an old person I do not become free to discuss my problems". (P10)

The clinic was reported not to be a friendly environment attributable to the nurse's attitudes. Adolescents expressed that once visiting the clinic after school, nurses told them that it's late, they are tired and they are knocking off. Even once they visit the clinic within the morning they will be asked plenty of questions like why you didn't go to school first.

Adolescents expressed that nurse's attitudes scare and discouraged them to go to the clinic, it additionally created them to hate the clinic. They reported that it is better to stay at home to avoid such kind of problems. Lastly, they indicated that they rather visit the clinic once they are seriously sick.

Based on the experiences participants suggested that nurses should implement what they said during health education throughout school health visit. Adolescents should be allocated a friendly young nurse, not rude nor judgmental. They need to additionally create services for youngsters to be friendly.

\subsubsection{The attitude of the Community Towards Adolescents}

The study found that adolescents experienced a negative attitude from elderly patients and people whom they know when visiting the clinic. Participants expressed that older people at the clinic believed that young people are not supposed to be sick. Elderly people additionally believed that adolescents are uncontrollable, do not have manners and pretence to be sick as a result they don't want to go to school. Participants additionally expressed that once they stumbled on a neighbour's or someone aware of them at the clinic they are going to be asked tons of queries and that they are being judged. As evidenced by:

"The reason I don't go to clinic when I am sick is that there are people at the clinic who are from where I stay so they will be judging me and also want to know why I'm sick. Some of my neighbours say hurtful words that I am sick because I don't respect my parents". (P5)

\subsubsection{Attitude and Influenced by Peers}

Adolescents expressed that once they are sick they initial consult their friends for recommendation before visiting the clinic. Many adolescents reported that another issue that contributes to delayed health-seeking behaviour is worry of being judged or ridiculed by peers. As one participant noted:

"Friends are judgmental as a result of last time after I told them I would like to go to the clinic they told me that I'm sick because I'm after boys and I'm a loose girl".(P1)

"Sometimes friends will pressurize you into doing things you do not like. Or sometimes you see your friends doing wrong things, but you end up willing to try those wrong things that they are doing just to feel what they are feeling. So yes, I can say girls of my age we have this thing called peer pressure". (P6)

Adolescents also explained that their friends associate visiting the clinic with being HIV positive; they reported that they believed that once youth visit the clinic they are infected with HIV and that they are attending to collect the treatment. As proven by:

"When you attend the clinic, they'll say you're sick, you're having HIV, and all stuff". (P4)

A 15-year-old adolescent reported that though the curriculum at school embrace health-related problems but the influence by peers has an additional impact on however they understand health care service utilization. As a 
participant noted:

"They teach us regarding teenage pregnancy, STI's, HIV and stuff, I will say most people as youth, once we are supposed to come to the clinic particularly there at schools, you may hear funny talks or rumours, however I will say, health is something that has got to be taken seriously". (P2)

Whereas, others indicated that school is the main aspect that stops them to go to the clinic because of spending most of their time at school and once they knock off the clinic is closed. They realize it tough to ask for permission at school to go to the clinic. As a 15-year-old participant noted:

"Most of the times I don't come to the clinic as a result of I'm in class throughout the day. Sometimes once I'm not in school I visit my family members at Mabopane, and I am a very secretive person. I find it very difficult to ask for permission at school or wherever to come to the clinic”. (P3)

Based on the experiences adolescents prompt that the nurses should to visit the school and educate learners concerning health problems, coupled with encouraging them to come to the clinic and not to share wrong information to other learners. As evidenced by:

"The nurses should go to school and educate learners about health issues, also encourage them to come to the clinic. They must also tell them not to judge others and not to spread wrong information to other learners". (P3)

\subsubsection{Knowledge of Health Information and Services Rendered for Adolescents at the Clinic}

Knowledge regarding health information amongst adolescents resulted in mixed feelings. Adolescents expressed positive as well as negative feelings regarding health issues. The negative feelings showed that adolescents appear to lack information about the complications, which could be caused by delayed health-seeking behaviours, and the services rendered for adolescents at the clinic. Lack of information was proven as a result of adolescents consulting their friends initial for recommendation once they are sick before visiting the clinic. Some reported to sleep and visit the clinic once seriously sick, however, they are aware that delays cloud lead to death. The participants indicated that their peers give them wrong information and myths concerning health issues and health services which scare them to visit the clinic. A 17-year-old adolescent in grade ten blames the shortage of life orientation teachers at school as the results of a lack of knowledge regarding health issues. The subsequent are excerpts shared by participants on the information of diseases and complication:

"I don't know anything about health issues because they do not teach us, we do not have a teacher for life orientation. The first thing that I do when I'm sick I sleep, and see what will happen”. (P4)

"Friends will tell you that if you utilize birth control it will create you unfertile or it'll harm your body, since then I don't consider to utilizing it". (P10)

"I solely visit the clinic once I'm seriously sick and that I know if I don't go to the clinic when I'm sick I'd die". (PI)

Participants who were pregnant found to book late for the antenatal clinic as a result of not being alert to the importance of early bookings. They indicated that their families failed to inform them. As a 19-year-old girl commented:

"The reason that I came to book late for Ante-natal clinic is that I didn't know that I need to book early and my family never told me to come and book". (P2)

Furthermore, most of the adolescents weren't alert to services rendered at the clinic that are youth-friendly they believed that a clinic may be a place you go once you are sick or people who are in chronic treatment. As a 15-year-old girl noted:

"The solely time I will be able to visit the clinic is once I'm sick and that I will never visit the clinic for other things. The clinic is for those that take period treatment and are near to die". (P4)

Whereas the other participants reported awareness regarding, peer pressure, family planning, HIV, uses of condom and teenage pregnancy as part of life orientation subject. As participants noted:

"They also teach us that we must use family planning so that we do not get pregnant. At school they teach us life orientation, usually; it's about HIV, condoms and teenage pregnancy”. (P6)

"Today they were teaching us about peer pressure at school saying, peer pressure does not exist but is something that we as people create for ourselves. We look at what other people do or have instead of minding our business. That is the opinion of those people who were teaching us. According to me, peer pressure exists “. (P6).

\subsection{Theme 2: The Logistical Aspects of Obtaining Care}

The study found that various barriers within and outside the clinic delays adolescents to seek health care. 


\subsubsection{Shortage of Treatment and Lack of Services Throughout Weekends}

In addition to the attitudes and influences, adolescents experienced concerns with regards to barriers faced in obtaining health services. Health services like birth control reported that is not available throughout weekends because the clinic solely attends only to emergencies. The weekend was reported to be the sole time that was convenient for adolescents to go to the clinic for those services. As a 17-year-old woman noted:

"...on weekends they are saying they deal with emergencies solely, so ah, is best simply just to stay at home". (P2)

Based on the experiences it was suggested that health care services should be available for adolescents throughout the weekends and after school. As evidenced by: "I think the nurses must help us fast when we come to the clinic and they must help us even on weekends and after school". (P1)

Some participants expressed considerations that there is a shortage of medication at the clinic and that they are taught to use remedies. The adolescents explained that there is no need for them to go to the clinic as some treatment for minor ailments is not out there. Some reported that they use over the counter medication like "grandpa" to treat their minor ailments. As a participant noted:

“...sometimes they will tell you that there is no treatment". (P1)

“...sometimes I become lazy to come to the clinic. I just buy Panado tablets at the shop and use them then I become better because even if I can come they will tell me that there is no medication, I must use remedies". (P8)

"Once I am sick I notify my parents at home and they give me medication, like grandpa". (P9)

\subsubsection{Long Queues and Waiting Time}

Long queues at the clinic found to be another barrier that prevents adolescents to seek health care. Many adolescents explained that they don't look for health as a result of the clinic is often full and nurses are slow in providing the services. There is no selected space at the clinic wherever adolescents will consult so that they are forced to face queue with all alternative clinic clients. It had been additionally indicated that consultation is sophisticated because the clients move from one cubicle to another for various procedures.

"First issue, those queues, yohh, additionally the very fact that everything is complicated, they take you from this side to that side and back to this site. You cannot find someone to help you directly". (P3)

"...the problem is that they will turn you back saying its late and the long queues that do not move". (P1)

Based on the experiences the participants suggested that adolescents should have their own queue and the nurse that focuses on them only.

"There must be a nurse who helps us as learners or maybe we must have our queue for consulting that would be fast". (P2)

\subsubsection{Distance and Family Responsibility}

Distance and lack of cash for the transport were expressed as a barrier that prevents few adolescents to access the clinic. Few participants indicated that they walk for a distance to access health care services, as a result of limited cash for transport. The participants explained that once they are seriously sick their parents hire non-public transport to the clinic. As evidenced by:

"I walk for about an hour to reach the clinic because I don't have cash for the taxi". (P5)

"...from school to here I walk almost 50 minutes it also depends on how fast you will be walking”. (P2)

"...from zone 3 to the clinic, I walk for more than an hour and a half". (P4)

As adolescents are, still below the care of the parents they are additionally expected to tell parents and caregivers before getting to the clinic, and generally are tough for them to disclose their wellness to the parents. As proven by:

"When I am sick firstly, I tell my mom then she will take me to the clinic or tell me to go on my own if I am not very sick, because she works far sometimes she tells my aunt to come and check me, but is not easy to tell them all my problems". (P1)

A 16-year-old girl said: I tell my grandmother first that I am sick; I tell her what is my problem and then maybe she will suggest that I go to the clinic. Sometimes I just stay at home, I'm a teen, I just think is one of those things that teenagers go through. Sometimes I am afraid to tell my grandmother, so I just keep quiet and hope it will pass".

Another issue was that other participant's parents are working far from home as a result adolescents are always busy with house chores and taking care of their siblings, they do not have time to visit the clinic. As evidenced by: 


\section{"My mum is working in Johannesburg, so after school, I cook, clean and do other things at home". (P1)}

\subsubsection{Common Reasons for Seeking Health}

The regular health care services utilized by adolescents in this study were family planning and antenatal care. Participants expressed that common reasons for visiting the clinic is for birth prevention and to book for antenatal care services. As participants noted: "I come to the clinic for booking for pregnancy (P3); "Most of the time I come for birth control pills". (P4)

\section{Discussion}

This paper explored and describe factors contributing to delay health-seeking behaviour amongst adolescents within the chosen clinic of North West province. These factors embrace the negative attitude of nurses, community, influenced by peers, shortage of treatment and lack of services throughout weekends, long queues, waiting time, distance and family responsibility, lack of knowledge concerning and towards health care systems and services rendered for adolescents at the clinic. These factors thus negatively affect adolescents' health-seeking behaviour. Being aware of factors contributing to delay health-seeking behaviour among adolescents will facilitate with setting future priorities to guard the health, well-being, and dignity of the adolescents.

Nurses and elderly people found to have a negative attitude towards adolescents seeking health care at Kutlwanong clinic. Nurse's negative attitude found to be the main issue that contributes to delay in health-seeking amongst adolescents. Similarly, staff stigma and therefore the unhealthy perceptions of nurses found to be factors that delayed men visiting the clinics in Durban, SA (Nyalela, Dlungwane, Taylor, \& Nkwanyana, 2018; Musinguzi et al., 2018). The National Department of Health (2011) in South Africa (SA) identified values and attitudes of staff as one of the six priorities areas that a lot of establishment has a lot of to enhance. The document indicates that health care providers are rude and uncaring to their patients. This drawback is highlighted through patient complaints and patient satisfaction surveys. Adolescents do not feel they have been treated well or in a caring manner at all times in this study. The National Core Standards for Health Institutions offer the general guide and measurements tools to quality care, however, the nurses in this clinic seem not to take into concern those systems in place to cut back the negative attitudes. Whereas elderly people had a perception that the clinic is for them not for young people. Adolescents who visit the clinic were labelled to possess bad manners and conducts. Elderly people believed that a well-behaved adolescent is healthy and must always be at school.

Waiting times and long queues are the intense explanation for dissatisfaction for adolescents attending the public clinic in this study. (Egbujie et al., 2018) indicated that long waiting times are a major source of dissatisfaction for patients attending public healthcare facilities in South Africa. Patient load per nurse, kind of service received and time of arrival in facilities were all associated with waiting times. The National Department of Health (2011) has identified waiting times as one of six vital areas for improvement. Health system-strengthening interventions to reinforce patient waiting time are being employed in public health facilities across SA as part of the 'Ideal Clinic' model. However, the interventions appear to not be effective, perhaps as a result of they're not well enforced at the clinic.

Shortages of medicines have been identified as another factor affecting health-seeking behaviours amongst adolescents. The results are kind of, as the study conducted within the European hospitals wherever ninety percent of respondents indicated that shortages of medicines are a current drawback in delivering the most effective care to patients (Miljković, Gibbons, Batista, Fitzpatrick, Underhill, \& Horák, 2019). Shortage of supplies and medicines in Uganda found to be a factor affecting compliance and health-seeking behaviour in hypertensive patients as mentioned by (Musinguzi et al., 2018). Supplier-related issues, lack of payment or failure to place orders with suppliers, failure to distribute medicine to health establishments and late ordering identified by the National Department of Health, (2011) in SA, Fast Track to quality document as the main reasons for this shortage.

Lack of services during weekends was also a concern to the adolescents as a result of weekends found to be a convenient time to visit the clinic. The results are just like the study conducted by (Bodenheimer \& Pham, 2010) within the USA that states that several patients who call their primary care practice throughout evenings or weekends were unable to get care. Access to health care is one of the patients' rights; therefore, governments ought to aim to provide universal and equitable access to high-quality health care services as explained by Scheffler, Visagie, and Schneider (2015).

The selected clinic was set in an exceedingly remote space, participants usually had to travel a long distance to reach the clinic. Most participants accessed the clinic on foot spending at least one hour on the way. Non-public cars are employed for very sick patients for those who stay away from the clinic. The price of transport was represented as a serious downside for health-seeking behaviour (Mattebo, Bogren, Brunner, Dolk, Pedersen, \& 
Erlandssonet, 2019), study unconcealed similar facts. Similarly, participants from rural communities of North West province found to possess a considerably lower obtainable weekly budget, not just for health care itself however additionally for transport to the health care facility (van der Hoeven et al., 2012). In other provinces of SA, like Western Cape accessibility to the health care facilities found to not be a problem as they lived within $3 \mathrm{~km}$ radius of the services (Scheffler et al., 2015).

Lack of knowledge may well be seen as a contributory factor, and as a reason why adolescents do not request health care services. Most adolescents in this study found to turn to friends or peers for information about health and disease. The findings Confirmed the limited access to health information, education and knowledge (Mattebo et al., 2019). Similarly, adolescent girls in Ghana found to lacks knowledge regarding health information like sexual and reproductive health (Kyilleh, Tabong, \& Konlaan, 2018). Availability of information to improve knowledge through school health services may be necessary for the development of appropriate health care services. In Uganda, regarding $81 \%$ of adolescents turned to parents, teachers, and other adults for information about health and disease. Whereas (56\%) browse a book/went to the library, (50\%) turned to siblings and friends and $(38 \%)$ indicated that they used the computer and Internet to search for health information. Younger respondents tended to rely upon siblings and friends for all types of health questions as mentioned by (Ybarra et al., 2007).

The mutual reason for visiting the clinic by adolescents was family planning and antenatal care. This finding is totally different to it of Otwombe et al., (2015) in Soweto wherever the explanations for seeking health was flu-like symptoms and also the one in Northern Republic of Finland was related to musculoskeletal pain as a results of being a member of a sports club. The findings in this study shows that there is a necessity for youth friendly services that specialize in development, gender, sexual orientation and reproductive health (Tanner, Philbin, Duval, Ellen, Kapogiannis, Fortenberry, \& Adolescent Trials Network for HIV/AIDS Interventions, 2014).

\section{Conclusion}

This study brings to light on the health-seeking behaviour of adolescent in a selected clinic of North West Province, SA. The results pointed to a set of factors delaying adolescent health-seeking behaviour. Thus, the findings can be considered for monitoring and evaluation of Adolescent and Youth Friendly Services to trace and measure the implementation of the Adolescents Health Youth Policy. The impacts of youth programs should be monitored regularly to assess progress according to the needs and capabilities of specific facilities. Compliance to the national core standards for health establishments should be ensured by the clinic manager to enhance the attitudes of nurses, waiting times and accessibility of medicine to satisfy adolescent's immediate expectations. Future research can investigate the adolescent's perceptions of how barriers to utilization of youth-friendly services are typically self-addressed in North West Province, SA.

\section{The Implications of the Study}

The current study reports factors contribute to delay health-seeking behaviour among adolescents in one of the rural setting community of Bojanala district, North West Province. Findings could be generalizable to a similar setting in Bojanala district. Given the fact that nurse's attitudes are reported as one of the foremost issues contributes to delay health care seeking behaviour, understanding the factors influencing nurse's behaviour amongst adolescents are a critical area of future research. Similarly, challenges faced by nurses in the clinic concerning compliance with the six ministerial priorities got to be explored. Moreover understanding the needs and challenges faced by the clinic with regards to the implementation of Youth Friendly Services is an important area for further inquiry.

\section{Limitations of the Study}

Our findings should be assessed amongst the constraints of the data. The focus of the study was to understand the factor, contributing to delayed health-seeking behaviour amongst adolescents better. As such, the aim, objectives and research questions should have been guided by one of the proposed frameworks that explain the behavioural aspects influencing utilization of health care services in an individual. These would have encouraged a broader perspective on the aspects of delaying adolescents to seek health care services. However, factors identified in this study relate to the characteristics of Andersen and Newman model (1973) and therefore the Kroeger's model (1983). Moreover, the researcher was in some cases asking leading questions which must be avoided in future research studies.

\section{Acknowledgments}

The authors would love to give thanks to all informants who participated during this study for sharing their views and knowledge. 


\section{Competing Interests Statement}

The author declared that there is no conflict of interest concerning the publication of my research work published at GSL.

\section{References}

Ajaegbu, O. O., \& Ubochi, I. I. (2016). Health Seeking Behaviour among Undergraduates in the Faculty of Health Sciences and Technology, University of Nigeria Enugu Campus. International Journal of Evaluation and Research in Education, 5(3), 181-188. https://doi.org/10.11591/ijere.v5i3.4537

Bodenheimer, T., \& Pham, H. H. (2010). Primary care: current problems and proposed solutions. Health Affairs, 29(5), 799-805. https://doi.org/10.1377/hlthaff.2010.0026

Channamma, R. (2017). Health care-seeking behavior. A theoretical perspective. Indian Journal of Research, 6(1), 790-792.

Creswell, J. W. (2014). Research Design: International student edition (4th ed.). London: Sage publications.

Dellany-Moretlwe, S., Cowan, F., Busza, J., Bolton-Moore, C., Kelley, K., \& Fairlie, L. (2015). Providing health services for young population: needs barriers and gaps. Journal of the International AIDS Society, 18, 19833. https://doi.org/10.7448/IAS.18.2.19833

Egbujie, B. A., Grimwood, A., Mothibi-Wabafor, E. C., Fatti, G., Tshabalala, A. M., Allie, S., \& Oyebanji, O. (2018). Impact of 'ideal clinic implementation on patient waiting time in primary healthcare clinics in KwaZulu-Natal Province. South African Medical Journal, 108(4), 311-318. https://doi.org/10.7196/SAMJ.2017.v108i4.12583

Fertman, C., Delgado, M., \& Tarasevich, S. (2014). Promoting child and adolescent mental care. Pennsylvania: University of Pittsburgh, Masters Dissertation.

James, S., Pisa, P., J, I., Beery, M., Martin, C., Skosana, C., \& Delany-Moretlwe, S. (2018). Assessment of adolescent and youth-friendly services in primary healthcare facilities in two provinces in South Africa. $B M C$ Health Service Research, 18, 809. https://doi.org/10.1186/s12913-018-3623-7

Jim, A. (2010). Health seeking behaviours in South Africa: a household perspective using the general households survey of 2007. Cape Town: (Doctoral dissertation, University of the Western Cape).

Kyilleh, J. M., Tabong, P. T., \& Konlaan, B. B. (2018). Adolescents' reproductive health knowledge, choices, and factors affecting reproductive health choices: a qualitative study in the West Gonja District in the northern region, Ghana. BMC interna. Ghana, BMC International Health and Human Rights, 18(1), 6. https://doi.org/10.1186/s12914-018-0147-5

Latunji, O. O., \& Akinyemi, O. O. (2018). Factors influencing health-seeking behaviour among civil servants in Ibadan, Nigeria. Annals of Ibadan Postgraduate Medicine, 16(1), 52-60.

Mattebo, M., Bogren, M., Brunner, N., Dolk, A., Pedersen, C., \& Erlandsson, K. (2019). (2019). Perspectives on adolescent girls' health-seeking behavior in relation to sexual and reproductive health in Nepal. Sexual Reproductive Healthcare, 20, 7-12. https://doi.org/10.1016/j.srhc.2019.01.006

Miljković, N., Gibbons, N., Batista, A., Fitzpatrick, R. W., Underhill, J., \& Horák, P. (2019). Results of EAHP's 2018 Survey on Medicines Shortages. Eur J Hosp Pharm, 26(2), 60-65. https://doi.org/10.1136/ejhpharm-2018-001835

Musinguzi, G., Anthierens, S., Nuwaha, F., Van Geertruyden, J. P., Wanyenze, R. K., \& Bastiaens, H. (2018). Factors influencing compliance and health-seeking behaviour for hypertension in Mukono and Buikwe in Uganda: a qualitative study. International Journal of Hypertension. https://doi.org/10.1155/2018/8307591

National Department of Health, D. (2011). Fast Track to Quality, the six most areas of patient-centered care. Tshwane: Department of Health.

National Department of Health, D. (2011). National Core Standards for Health Establishment in South Africa. Tshwane: Department of Health.

National Department of Health, D. (2017). 2017 National Adolescent \& Youth Health Policy. Tshwane: Department of Health.

Nyalela, M., Dlungwane, T., Taylor, M., \& Nkwanyana, N. (2018). Health seeking and sexual behaviour of men presenting with sexually transmitted infections in two primary health care clinics in Durban. Southern African 
Journal of Infectious Diseases, 1-6. https://doi.org/10.1080/23120053.2018.1520480

Otwombe, K., Dietrich, J., Laher, F., Hornschuh, S., Nkala, B., Chimoyi, L., \& Miller, C. L. (2015). Health-seeking behaviours by gender among adolescents in Soweto, South Africa. Global Health Action, 8(1), 25670. https://doi.org/10.3402/gha.v8.25670

Scheffler, E., Visagie, S., \& Schneider, M. (2015). The impact of health service variables on healthcare access in a low resourced urban setting in the Western Cape, South Africa. African Journal of Primary Health Care \& Family Medicine, 1, 1-11. https://doi.org/10.4102/phcfm.v7i1.820

Tanner, A. E., Philbin, M. M., Duval, A., Ellen, J., Kapogiannis, B., Fortenberry, J. D., \& Adolescent Trials Network for HIV/AIDS Interventions. (2014). "Youth friendly" clinics: Considerations for linking and engaging HIV-infected adolescents into care. AIDS Care, 26(2), 199-205. https://doi.org/10.1080/09540121.2013.808800

Tesfaye, G., Chojenta, C., Smith, R., \& Loxton, D. (2018). Application of the Andersen-Newman model of health care utilization to understand antenatal care use in Kersa District, Eastern Ethiopia. PloS one, 13(12), e0208729. https://doi.org/10.1371/journal.pone.0208729

van der Hoeven, M., Kruger, A., \& Greeff, M. (2012). Differences in health care seeking behaviour between rural and urban communities in South Africa. International Journal for Equity in Health, 1, 31. https://doi.org/10.1186/1475-9276-11-31

Ybarra, M. L., Emenyonu, N., Nansera, D., Kiwanuka, J., \& Bangsberg, D. R. (2007). Health information-seeking among Mbararan adolescents: results from the Uganda Media and You survey. Health Education Research, 23(2), 249-258. https://doi.org/10.1093/her/cym026

\section{Copyrights}

Copyright for this article is retained by the author(s), with first publication rights granted to the journal.

This is an open-access article distributed under the terms and conditions of the Creative Commons Attribution license (http://creativecommons.org/licenses/by/4.0/). 